\title{
miR-34a targets BCL-2 to suppress the migration and invasion of sinonasal squamous cell carcinoma
}

\author{
YIGANG ZHAO and XIANZHI WANG \\ Department of Otolaryngology, Linyi People's Hospital, Linyi, Shandong 276000, P.R. China
}

Received March 6, 2018; Accepted August 28, 2018

DOI: $10.3892 / 01.2018 .9427$

\begin{abstract}
Sinonasal squamous cell carcinomas (SN-SCC) are rare tumors with low survival rate. It was reported that miR-34a expression is low in many cancers and acted as a tumor suppressor. But the biological function of miR-34a in SN-SCC has hardly been reported. Therefore, we explored the role and underlying mechanism of miR-34a in the migration and invasion of SN-SCC. Western blot analysis and RT-PCR were carried out to examine B-cell lymphoma-2 (BCL-2) and miR-34a expression in SN-SCC. Transwell assay was performed to test the SN-SCC migratory and invasive ability. Luciferase reporter assay was carried out to verify the target of miR-34a. Results demonstrated that miR-34a expression was lower in SN-SCC tissues and cells than normal SN-SCC. Re-expression of miR-34a inhibited cell migration and invasion, while had the opposite effect on inhibition of miR-34a. We also found that BCL-2 expression was higher in SN-SCC and silencing BCL-2 curbed the development of SN-SCC. BCL-2 was found to be a target of miR-34a and negatively correlated with miR-34a expression. Furthermore, BCL-2 attenuated the miR-34a inhibitory effect on SN-SCC cell migration and invasion. In short, these data demonstrated that miR-34a inhibited SN-SCC cell migration and invasion through targeting BCL-2.
\end{abstract}

\section{Introduction}

Sinonasal squamous cell carcinoma (SN-SCC) is a malignant tumor derived from the respiratory epithelium of the sinonasal cavity, which accounts for approximately 3-6\% of all head and neck cancers $(1,2)$. SN-SCC appears mainly in nasal cavity and approximately $30 \%$ of the patients are related to contact with leather, textile or wood $(3,4)$. Currently, the best treatment is postoperative radiotherapy, accompanied by adjuvant chemotherapy. Local recurrence of SN-SCC is the main reason for low survival rate $(5,6)$. Therefore, looking for the additional

Correspondence to: Dr Xianzhi Wang, Department of Otolaryngology, Linyi People's Hospital, 27 Jiefang Street, Linyi, Shandong 276000, P.R. China

E-mail: xe94hx@163.com

Key words: miR-34a, migration, invasion, SN-SCC, DAB2 options for treatment of SN-SCC and knowing the underlying mechanism of SN-SCC progression is necessary.

miRNAs have been reported to regulate gene expression by binding to the 3'-UTR mRNA (7). Many miRNAs are thought to be tissue-specific and the expression varies in different human cancer cells. They regulate tumor cell development, such as lung, ovary, breast cancer and cancer of the esophagus (8-11). However, there are fewer results showing the role of miRNAs in head and neck cancer including SN-SCC.

Abundant evidence shows that miR-34a expression was lower in tumors and showed an inhibitory effect on the progression of various cancers. For example, miR-34a exhibited suppression effect on esophageal squamous cell carcinoma via regulating PLCE1 (12) and inhibited the progress of gastric cancer via regulating HK1 (13). Also, miR-34a mimic inhibited ovarian cancer and cervical cancer cell proliferation by targeting BCL-2 $(14,15)$, whereas inhibiting miR-34a promoted colorectal cancer and lung cancer development $(16,17)$. A study found that miR-34a expression was lower in SN-SCC and related to the poor prognosis of patients (18). However, the biological function of miR-34a in SN-SCC progression and whether miR-34a targeted BCL-2 in regulating SN-SCC migration and invasion has not been previously reported.

B-cell lymphoma-2 (BCL-2) plays an important role in cell apoptosis. In all tumors, apoptosis is an important target for treatment intervention. Previous studies had reported that BCL-2 induced apoptosis of mitochondrial cells, indicating that BCL-2 played a role in tumor development by blocking apoptosis (19). BCL-2 expression was higher in multiple cancers and regulated by various miRNAs, such as pancreatic adenocarcinoma regulated by miR-126 (20), breast cancer regulated by miR-27a (21), gastric cancer by miR-711 (22), pancreatic cancer by miR-1180 (23). A previous study reported that $\mathrm{BCL}-2$ expression was obviously increased in SN-SCC $(24,25)$ and it is involved in the tumor cell proliferation, migration, invasion and apoptosis. However, the biological mechanism of BCL-2 in SN-SCC cell regulated by miR-34a has not been reported.

Here, we studied the potential links of miR-34a and SN-SCC. Firstly, we found a low expression of miR-34a in SN-SCC cells and tissues by RT-PCR, suggesting miR-34a inhibitory effect in SN-SCC. Secondly, miR-34a inhibited SN-SCC cell migration and invasion. Thirdly, we found that the potential mechanism of miR-34a in SN-SCC was achieved 
by targeting BCL-2. We finally found that inhibitory effect of miR-34a in SN-SCC could be attenuated by BCL-2. Our research indicated that miR-34a/BCL-2axis was a potential target for treating SN-SCC.

\section{Materials and methods}

SN-SCC samples. Fifty-two SN-SCC specimens were obtained from patients who underwent surgery at Linyi People's Hospital (Linyi, China) from 2011 to 2017. Experienced pathologists confirmed the tumor and normal tissues through HE staining. Finally, tissues were stored in a refrigerator at $-80^{\circ} \mathrm{C}$. The study was approved by the Ethics Committee of Linyi People's Hospital. Signed written informed consents were obtained from the patients or guardians.

Cell line establishment and cell transfection. All SN-SCC cell lines (RPMI-2650, SCCNC2 and SCCNC7) used in this study were derived from the previously untreated primary SCC, which originated in the maxillary sinus. Cells were cultured in DMEM, containing 10\% FBS, penicillin (100 U/ml) and streptomycin $(100 \mu \mathrm{g} / \mathrm{ml})$ (Beijing Solarbio Science \& Technology Co., Ltd., Beijing, China), incubated at $37^{\circ} \mathrm{C}$ with $5 \% \mathrm{CO}_{2}$.

Synthetic miR-34a mimic/inhibitor was provided by GenePharma Co., Ltd. (Shanghai, China). We transfected miR-34a mimic into SN-SCC cells to overexpress miR-34a or miR-34a inhibitor to silence miR-34. SCCNC2 and SCCNC7 cells were added into 24 -well plates $24 \mathrm{~h}$ before transfection. The Lipofectamine $2000^{\mathrm{TM}}$ reagent (Invitrogen; Thermo Fisher Scientific, Inc., Carlsbad, CA, USA) was used to perform the transfection into SN-SCC cell lines the next day. The transfected cells were divided into several groups.

Transwell assay. The cells of each group were digested at the logarithmic stage of growth and the number of cells was calculated after transfected with miR-34a for $48 \mathrm{~h}$. We seeded SN-SCC cells $\left(1 \times 10^{5}\right.$ cells) onto the upper chamber membrane with the medium being serum free. The RPMI-1640 medium contained $20 \%$ fetal calf serum was seeded into the lower chamber as a chemoattractant and incubated for $24 \mathrm{~h}$ at $37^{\circ} \mathrm{C}$. Then, the upper chamber cells migrated into the lower chamber and $0.1 \%$ crystal violet was used to stain the migrated cells for another $30 \mathrm{~min}$, eventually, images of the migration cells in eight random views were photographed under a microscope (Olympus, Tokyo, Japan) for records. For invasion assay, except for coating the filter in the upper chamber with Matrigel, it was similar to the Transwell migration assay.

Reverse Transcription-quantitative PCR (RT-qPCR). TRIzol reagent (Invitrogen; Thermo Fisher Scientific, Inc.) was used to extract total RNA and Nanodrop 1000 (Thermo Fisher Scientific, Inc.) was carried out to quantify RNA expression. The stem-loop real-time PCR miRNA kit (Guangzhou RiboBio Co., Ltd., Guangzhou, China) was carried out to perform the specific reverse transcription. SYBR Premix Ex Taq II (Takara Bio, Inc., Otsu, Japan) was used to perform quantitative PCR. U6 and GAPDH were used as an internal control. The $2^{-\Delta \Delta \mathrm{Cq}}$ method was used to detect the mRNA expression. The sequences of the primers used were: miR-34a-F: GCGGCCAATCAGCAAGTATACT, and R: GTG
CAGGGTCCGAGGT. BCL-2-F: GAAGCACAGATGGTT GATGC, and R: CACCTCACAAGGTTCCAAT. U6-F: ATT GGAACGATACAGAGAAGATT, and R: GGAACGCTT CACGAATTTG.GAPDH-F:TGGTATCGTGGAAGGACTC, and R: AGTAGAGGCAGGGATGATG.

Western blot analysis. RIPA lysis buffer containing proteinase inhibitors (Beyotime Institute of Biotechnology, Haimen, China) were used to extract total protein from the SN-SCC cells or tissues. Protein concentration measured using BCA reagent kit (Beyotime Institute of Biotechnology). We analyzed the amount of protein by using immunoblotting method. Protein in each group $(50 \mathrm{mg}$ ) was added into the well of polyacrylamide gel and separated by $10 \%$ SDS-PAGE and then transferred to nitrocellulose filter (NC) membrane (EMD Millipore, Billerica, MA, USA). Then, skim milk (5-10\%) was used to block the membrane at room temperature for $2 \mathrm{~h}$ and incubated with the primary antibodies: Bcl-2 (10C4) mouse monoclonal IgG1, cat. no. sc-23960, 1:500 from Santa Cruz Biotechnology, Inc. (Dallas, TX, USA). GAPDH (D16H11) XP ${ }^{\circledR}$ Rabbit mAb; cat. no. 5174; 1:1,000; Cell Signaling Technology, Inc. (Danvers, MA, USA) followed by the secondary antibodies: Goat anti-rabbit IgG-HRP, cat. no. sc-2004; 1:2,000 and goat anti-mouse IgG-HRP; cat. no. sc-2005; 1:2,000 (both from Santa Cruz Biotechnology, Inc.). Subsequently, the membrane was detected using an ECL chemiluminescence detection kit. GAPDH served as the loading control.

Dual luciferase reporter assay. The relative luciferase ability was performed using the recombinant pMIR-REPORT luciferase vector. The wild-type and mut-type BCL-2 3'UTR were constructed downstream of pMIR-REPORT luciferase vector. We used Lipofectamine 2000 to transfect SN-SCC cells with control mimic and miR-34a mimic. The dual luciferase reporter assay system (Promega Corporation, Madison, WI, USA) was then used to measure the luciferase activity values.

Statistical analysis. All independent experiments were conducted three times. Data are presented as mean \pm SD. SPSS 19.0 software (IBM Corp., Armonk, NY, USA) and GraphPad Prism 5.02 Software (GraphPad Software, Inc., La Jolla, CA, USA) were used to perform statistical analysis and complete graph presentations, respectively. One-way analysis of variance (ANOVA) was used and the post hoc test was Tukey's post hoc, to compare the differences of the groups. The correlation was analyzed by Pearson's regression analysis. $\mathrm{P}<0.05$ was considered to indicate a statistically significant difference.

\section{Results}

miR-34a was overexpressed and PAQR3 was lower expressed in $S N-S C C$. miR-34a and BCL-2 expression in 52 paired SN-SCC tissues and normal tissues was detected by RT-PCR. As Fig. 1A and $\mathrm{C}$ shows, the miR-34a average expression in SN-SCC tissues was reduced markedly, while BCL-2 expression was increased obviously. We then analyzed miR-34a expression in SN-SCC cells: RPMI-2650, SCCNC2 and SCCNC7 cells. miR-34a expression was decreased in all cells while PAQR3 was increased (Fig. 1B and D), which was 

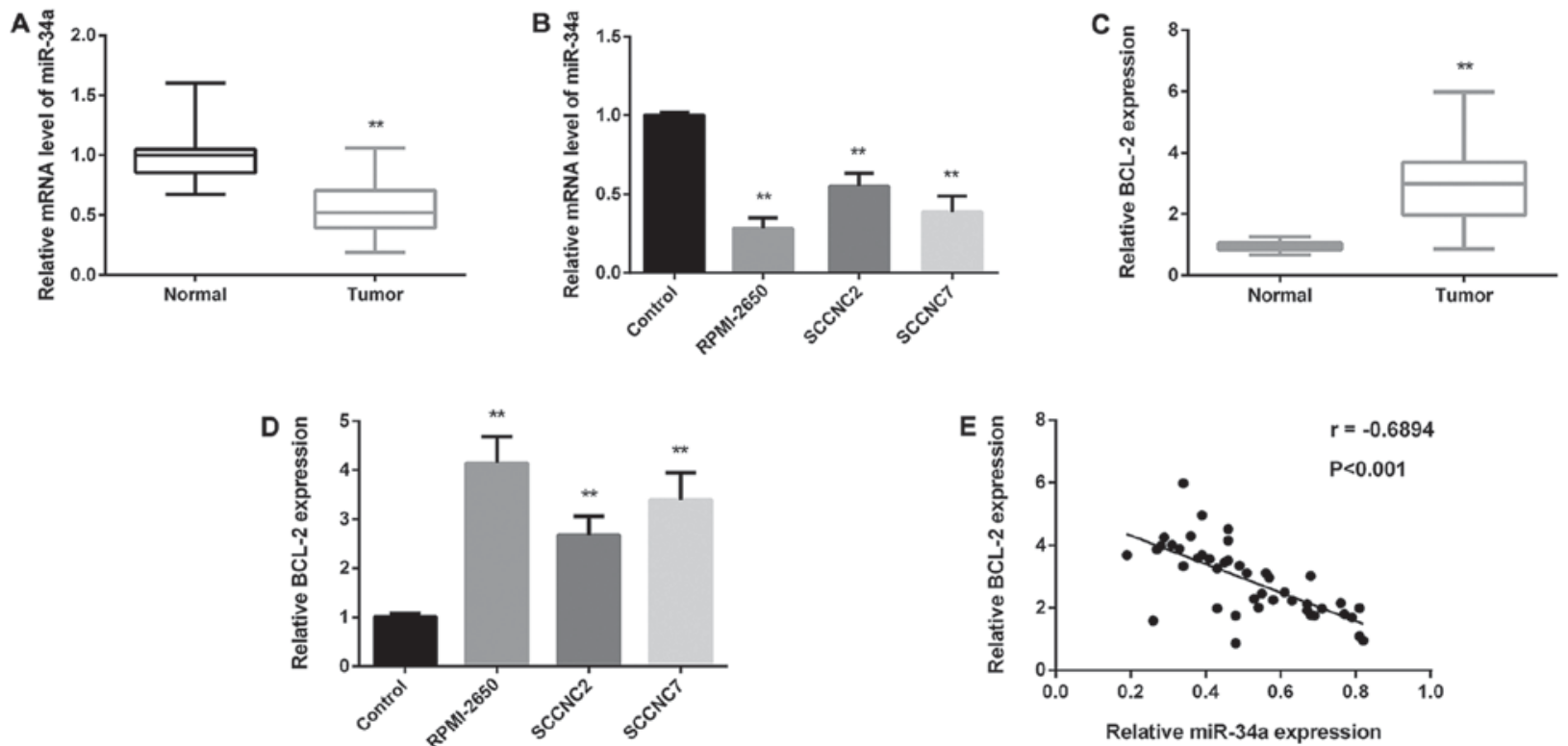

Figure 1. Low expression of miR-34a and high expression of BCL-2 in SN-SCC. (A) Detection of miR-34a level in SN-SCC tissues by RT-PCR. (B) Detection of miR-34a level in SN-SCC cell lines RPMI-2650, SCCNC2 and SCCNC7. (C) Detection of BCL-2 level in SN-SCC tissues by RT-PCR. (D) Detection of BCL-2 level in SN-SCC cell lines RPMI-2650, SCCNC2 and SCCNC7. (E) The negative correlation of miR-34a and BCL-2 expression ( $\mathrm{r}=-0.6894, \mathrm{P}<0.001)$ as determined by Pearson's regression analysis. ${ }^{* *} \mathrm{P}<0.01$. BCL-2, B-cell lymphoma-2; SN-SCC, sinonasal squamous cell carcinoma.

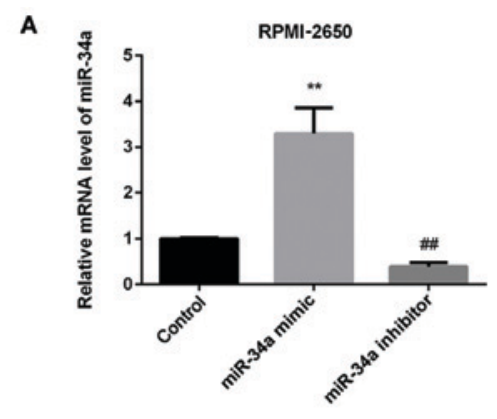

B

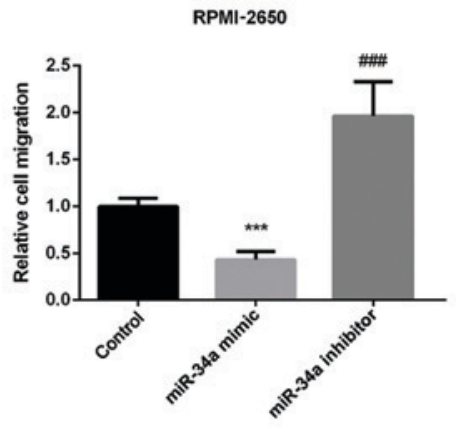

C

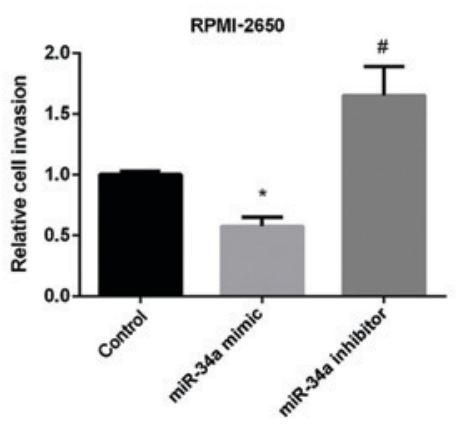

Figure 2. Inhibition effect of miR-34a on SN-SCC cells migration and invasion. (A) miR-34a expression detected in RPMI-2650 cells by western blot analysis and RT-PCR after treated with miR-34a mimic or inhibitor. (B) Relative cell migration detected in RPMI-2650 cells after treated with miR-34a mimic or inhibitor. (C) Relative cell invasion detected in RPMI-2650 cells after treated with miR-34a mimic or inhibitor. ${ }^{*} \mathrm{P}<0.05,{ }^{* * *} \mathrm{P}<0.01,{ }^{* * * *} \mathrm{P}<0.001 ;{ }^{*} \mathrm{P}<0.05$, ${ }^{\#} \mathrm{P}<0.01,{ }^{\# \#} \mathrm{P}<0.001$. SN-SCC, sinonasal squamous cell carcinoma. 
A
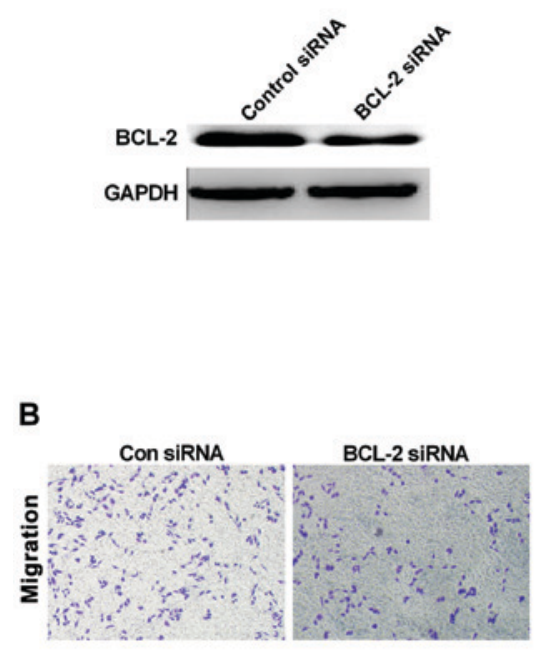

C

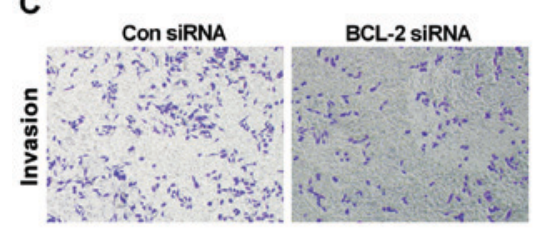

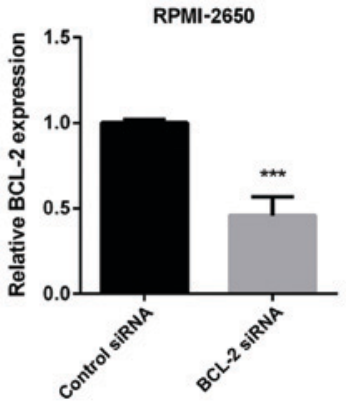

RPMI-2650

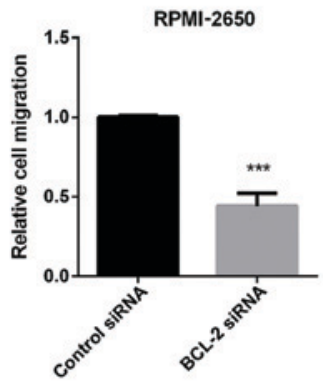

RPMI-2650

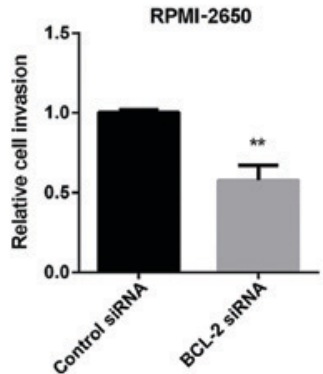

Figure 3. Inhibition effect of BCL-2 siRNA on migration and invasion of SN-SCC cells. (A) Transfection efficiency of BCL-2 siRNA detected in RPMI-2650 cells. (B) Migration of RPMI-2650 cells in con siRNA and BCL-2 siRNA group. (C) Invasion of RPMI-2650 cells in con siRNA and BCL-2 siRNA group. ${ }^{* * *} \mathrm{P}<0.01,{ }^{* * * *} \mathrm{P}<0.001$. BCL-2, B-cell lymphoma-2; SN-SCC, sinonasal squamous cell carcinoma.

A

$\begin{array}{cc}\text { 203-209 of BCL2 3'-UTR } & \text {... UCGAAUCAGCUAUUU-ACUGCCAA... } \\ \text { IIIIIII } \\ \text { hsa-miR-34a } & \ldots \text { UGUUGGUCGAUUCUGUGACGGU... }\end{array}$

C

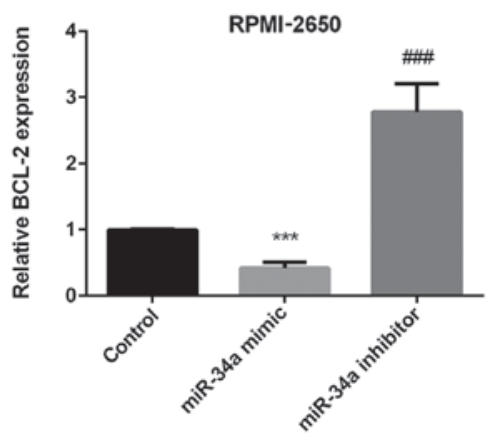

B

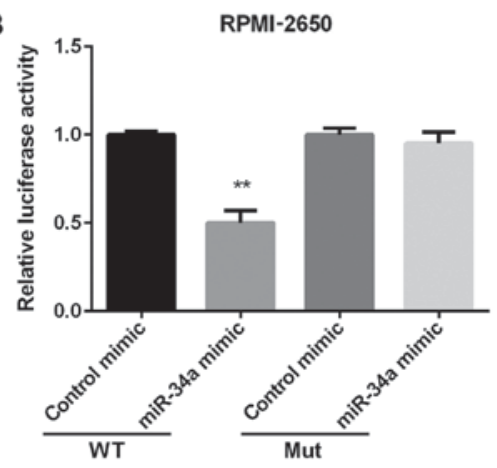

Figure 4. Confirmation of BCL-2 as the target gene of mir-34a in SN-SCC. (A) Display of the binding site of BCL-2 and miR-34a. (B) The luciferase activities in RPMI-2650 cells transfected with BCL-2-3'-UTR-wild (WT) or BCL-2-3'-UTR- mutant (Mut). (C) Relative BCL-2 mRNA and protein expression using RT-PCR and western blot analysis in RPMI-2650 cells. ${ }^{* *} \mathrm{P}<0.01 ;{ }^{* * * *} \mathrm{P}<0.001 ;{ }^{* \# \#} \mathrm{P}<0.001$. BCL-2, B-cell lymphoma-2; SN-SCC, sinonasal squamous cell carcinoma. 
A

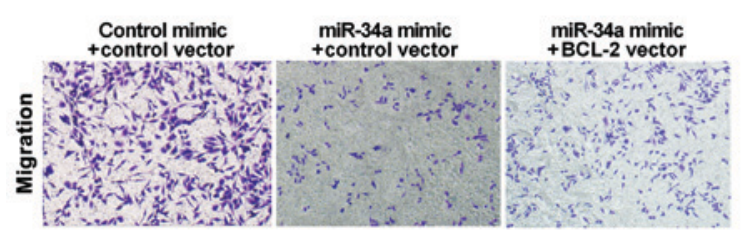

B

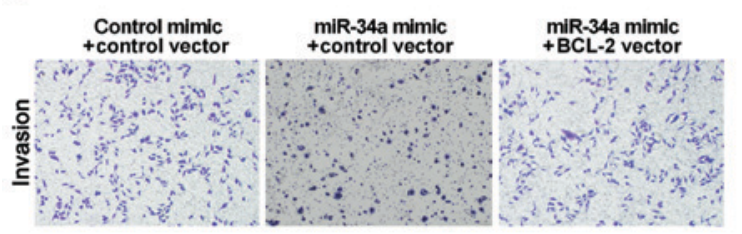

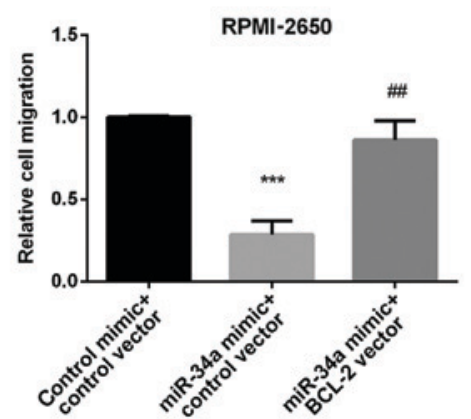

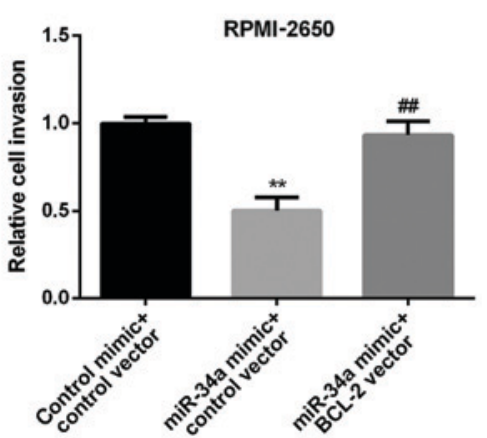

Figure 5. The inhibition effect of miR-34a in SN-SCC reversed by BCL-2. (A) Comparison of the migration of RPMI-2650 cells after overexpression of miR-34a alone or both miR-34a and BCL-2. (B) Comparison of the invasion of RPMI-2650 cell overexpression of miR-34a alone or both miR-34a and BCL-2. ${ }^{* *} \mathrm{P}<0.01,{ }^{* * *} \mathrm{P}<0.001 ;{ }^{\# \#} \mathrm{P}<0.01$. SN-SCC, sinonasal squamous cell carcinoma; BCL-2, B-cell lymphoma-2.

similar to SN-SCC tissues. Based on the results above, we further analyzed the correlation between miR-34a and PAQR3 expression, as Pearson's regression analysis showed that they were negatively correlated (Fig. 1E).

miR-34a suppressed $S N-S C C$ cell migration and invasion. We then investigated miR-34a effect on SN-SCC. Firstly, we overexpressed or silenced miR-34a through transfecting miR-34a mimic or inhibitor into RPMI-2650 cells. As seen in Fig. 2A, the transfection was successful. Secondly, we used Transwell assay to detect how miR-34a affected SN-SCC cell migration and invasion and found that upregulation of miR-34a inhibited SN-SCC cell migration, whereas knockdown of miR-34a had the opposite effect (Fig. 2B). Fig. 2C shows inhibitory effect of cell invasion by miR-34a mimic and promotion effect by miR-34a inhibitor in SN-SCC.

Silencing BCL-2 curbs the development of $S N-S C C$. As previous study proved that BCL-2 was overexpressed in SN-SCC, we chose to silence the expression of BCL-2 to explore the role of BCL-2 in SN-SCC. The efficiency of silencing BCL-2 is shown in Fig. 3A. Then, we examined the relative cell migration and invasion when silencing BCL-2 using Transwell assay. As the results show, BCL-2 siRNA inhibited SN-SCC cell migration and invasion (Fig. 3B and C).

BCL-2 was verified as the target of miR-34a in SN-SCC. As previous reported BCL-2 functioned as a tumor promoter and targeted by miR-34a in osteosarcoma. We suspected that miR-34a targeted BCL-2 in regulating SN-SCC. We used TargetScanHuman 7.1 to validate this prediction. The binding sites of BCL-2 and miR-34a are presented in Fig. 4A. Then, we used luciferase reporter assay to detect the luciferase ability in RPMI-2650 cells to further determine the accuracy of this prediction. The results showed that miR-106b mimic group had a significantly reduced luciferase activity compared to control group in wild-type, whereas, there was no effect in mut-type in RPMI-2650 cells (Fig. 4B). Finally, we detected whether miR-34a could affect BCL-2 expression in SN-SCC cells. As shown in Fig. 4C, miR-34a mimic decreased both miR-34a mRNA and protein level, while increased by miR-34a inhibitor in RPMI-2650 cells.

$B C L-2$ reverses the inhibitory effect of miR-34a in $S N-S C C$. We explored whether BCL-2 could affect the miR-34a on SN-SCC cell migration and invasion. We divided the cells into three groups: Control, miR-34a mimic and miR-34a mimic + BCL-2 vector. As the results show, the migrated cells of RPMI-2650 were increased when co-overexpression of both BCL-2 and miR-34a compared with the cells overexpressied with miR-34a alone (Fig. 5A). Also, the same effect of the invasive cells of RPMI-2650 was found (Fig. 5B). These data indicated that BCL-2 could reverse the inhibitory effect of miR-34a in regulating SN-SCC cell invasion and migration.

\section{Discussion}

SN-SCC is a common malignant tumor. The occurrence and progression of SN-SCC are not only related to abnormal proliferation of cells, but are also associated with the abnormal apoptosis.

miR-34a was proved to express abnormally in multiple cancers and to act as a tumor inhibitor (26). Increasing evidence stated that miR-34a had an inhibitory effect on 
tumor progression, such as inhibiting medulloblastoma cell proliferation and promoting cell apoptosis. Moreover, it had the same effect on gastric cancer (27), prostate cancer (28), hepatocellular carcinoma cells (29), head and neck squamous cell carcinoma (30), laryngeal squamous cell carcinoma (31). Our study showed a decreased expression of miR-34a in SN-SCC and inhibited cell migratory and invasive ability, which is consistent with a previous study that miR-34a expression was lower in SN-SCC and associated with the poor prognosis of patients (18).

BCL-2 acted as an apoptosis factor and was proven to take part in regulating some cancers targeted by miR-34a. It was reported by Wen et al that miR-34a inhibited osteosarcoma cell invasion and migration via targeting BCL-2 and C-IAP2 (32), which is similar to the study that miR-34a suppressed the viability and migration of breast cancer by inhibiting BCL-2 (33). Wang et al showed that miR-34a mimic curbed cell viability and facilitated the apoptosis of cervical cancer cell through targeting BCL-2 (15). In our study, we found that miR-34a curbed SN-SCC cell migratory and invasive ability by targeting BCL-2.

In conclusion, miR-34a expression was lower while BCL-2 was higher in SN-SCC and their correlation was negative. We proved for the first time that BCL-2 was a direct target of miR-34a in regulating the progress of SN-SCC and BCL-2 could attenuate miR-34a inhibition effect on SN-SCC, indicating that miR-34a/BCL-2 axis has potential application value in $\mathrm{SN}-\mathrm{SCC}$ diagnosis and therapy.

\section{Acknowledgements}

Not applicable.

\section{Funding}

No funding was received.

\section{Availability of data and materials}

The datasets used and/or analyzed during the present study are available from the corresponding author on reasonable request.

\section{Authors' contributions}

YZ designed this study and collected the data. YZ and XW performed the experiments and interpreted the results. XW wrote and finalized the manuscript. Both authors read and approved the final manuscript.

\section{Ethics approval and consent to participate}

The study was approved by the Ethics Committee of Linyi People's Hospital (Linyi, China). Patients who participated in this research had complete clinical data. Signed written informed consents were obtained from the patients or guardians.

\section{Patient consent for publication}

Not applicable.

\section{Competing interests}

The authors declare that they have no competing interests.

\section{References}

1. Ansa B, Goodman M, Ward K, Kono SA, Owonikoko TK, Higgins K, Beitler JJ, Grist W, Wadsworth T, El-Deiry M, et al: Paranasal sinus squamous cell carcinoma incidence and survival based on Surveillance, Epidemiology, and End Results data, 1973 to 2009. Cancer 119: 2602-2610, 2013.

2. Turner JH and Reh DD: Incidence and survival in patients with sinonasal cancer: A historical analysis of population-based data. Head Neck 34: 877-885, 2012.

3. Bonzini M, Battaglia P, Parassoni D, Casa M, Facchinetti N, Turri-Zanoni M, Borchini R, Castelnuovo P and Ferrario MM: Prevalence of occupational hazards in patients with different types of epithelial sinonasal cancers. Rhinology 51: 31-36, 2013.

4. Sanghvi S, Khan MN, Patel NR, Yeldandi S, Baredes S and Eloy JA: Epidemiology of sinonasal squamous cell carcinoma: A comprehensive analysis of 4994 patients. Laryngoscope 124: 76-83, 2014.

5. Dulguerov P and Allal AS: Nasal and paranasal sinus carcinoma: How can we continue to make progress? Curr Opin Otolaryngol Head Neck Surg 14: 67-72, 2006.

6. Youlden DR, Cramb SM, Peters S, Porceddu SV, Møller H, Fritschi L and Baade PD: International comparisons of the incidence and mortality of sinonasal cancer. Cancer Epidemiol 37: 770-779, 2013.

7. Scaria V, Hariharan M, Pillai B, Maiti S and Brahmachari SK: Host-virus genome interactions: Macro roles for microRNAs. Cell Microbiol 9: 2784-2794, 2007.

8. Zhu W, Shan X, Wang T, Shu Y and Liu P: miR-181b modulates multidrug resistance by targeting BCL 2 in human cancer cell lines. Int J Cancer 127: 2520-2529, 2010.

9. Yang H, Kong W, He L, Zhao JJ, O'Donnell JD, Wang J, Wenham RM, Coppola D, Kruk PA, Nicosia SV, et al: MicroRNA expression profiling in human ovarian cancer: miR-214 induces cell survival and cisplatin resistance by targeting PTEN. Cancer Res 68: 425-433, 2008.

10. Pogribny IP, Filkowski JN, Tryndyak VP, Golubov A, Shpyleva SI and Kovalchuk O: Alterations of microRNAs and their targets are associated with acquired resistance of MCF-7 breast cancer cells to cisplatin. Int J Cancer 127: 1785-1794, 2010.

11. Hamano R, Miyata H, Yamasaki M, Kurokawa Y, Hara J, Moon JH, Nakajima K, Takiguchi S, Fujiwara Y, Mori M, et al: Overexpression of miR-200c induces chemoresistance in esophageal cancers mediated through activation of the Akt signaling pathway. Clin Cancer Res 17: 3029-3038, 2011.

12. Cui XB, Peng H, Li RR, Mu JQ, Yang L, Li N, Liu CX, Hu JM, Li SG, Wei Y, et al: MicroRNA-34a functions as a tumor suppressor by directly targeting oncogenic PLCE1 in Kazakh esophageal squamous cell carcinoma. Oncotarget 8: 92454-92469, 2017.

13. Zhou Y, Ding BZ, Lin YP and Wang HB: miR-34a, as a suppressor, enhance the susceptibility of gastric cancer cell to luteolin by directly targeting HK1. Gene 644: 56-65, 2018.

14. Ding N, Wu H, Tao T and Peng E: NEAT1 regulates cell proliferation and apoptosis of ovarian cancer by miR-34a-5p/BCL2. OncoTargets Ther 10: 4905-4915, 2017.

15. Wang X, Xie Y and Wang J: Overexpression of microRNA-34a-5p inhibits proliferation and promotes apoptosis of human cervical cancer cells by downregulation of Bcl-2. Oncol Res: Aug 30, 2017 (Epub ahead of print).

16. Zhang X, Ai F, Li X, Tian L, Wang X, Shen S and Liu F: MicroRNA-34a suppresses colorectal cancer metastasis by regulating Notch signaling. Oncol Lett 14: 2325-2333, 2017.

17. Li Y, Zhang H, Dong Y, Fan Y, Li Y, Zhao C, Wang C, Liu J, Li X, Dong M, et al: miR-146b-5p functions as a suppressor miRNA and prognosis predictor in non-small cell lung cancer. $\mathrm{J}$ Cancer 8: 1704-1716, 2017.

18. Ogawa T, Saiki Y, Shiga K, Chen N, Fukushige S, Sunamura M, Nagase H, Hashimoto S, Matsuura K, Saijo S, et al: miR-34a is downregulated in cis-diamminedichloroplatinum treated sinonasal squamous cell carcinoma patients with poor prognosis. Cancer Sci 103: 1737-1743, 2012.

19. Xiong S, Zheng Y, Jiang P, Liu R, Liu X and Chu Y: MicroRNA-7 inhibits the growth of human non-small cell lung cancer A549 cells through targeting BCL-2. Int J Biol Sci 7: 805-814, 2011. 
20. Feng SD, Mao Z, Liu C, Nie YS, Sun B, Guo M and Su C: Simultaneous overexpression of miR-126 and miR-34a induces a superior antitumor efficacy in pancreatic adenocarcinoma. OncoTargets Ther 10: 5591-5604, 2017.

21. Wu J, Sun Z, Sun H and Li Y: MicroRNA 27a promotes tumorigenesis via targeting AKT in triple negative breast cancer. Mol Med Rep 17: 562-570, 2018.

22. Liao A, Tan G, Chen L, Zhou W and Hu H: RASSF1A inhibits gastric cancer cell proliferation by miR-711- mediated downregulation of CDK4 expression. Oncotarget 7: 5842-5851, 2016.

23. Gu L, Zhang J, Shi M and Peng C: The effects of miRNA-1180 on suppression of pancreatic cancer. Am J Transl Res 9: 2798-2806, 2017.

24. Lu H, Wang C, Hao L, Yin G and Hao R: The expression and significance of programmed cell death 5 and B-cell lymphoma/leukemia-2 in sinonasal squamous cell carcinoma. Lin Chung Er Bi Yan Hou Tou Jing Wai Ke Za Zhi 28: 13011304, 2014 (In Chinese).

25. Katori H, Nozawa A and Tsukuda M: Cell proliferation, apoptosis, and apoptosis inhibition in malignant transformation of sinonasal inverted papilloma. Acta Otolaryngol 127: 540-546, 2007.

26. Cao W, Yang W, Fan R, Li H, Jiang J, Geng M, Jin Y and Wu Y: miR-34a regulates cisplatin-induce gastric cancer cell death by modulating PI3K/AKT/survivin pathway. Tumour Biol 35: 1287-1295, 2014.

27. Cao W, Fan R, Wang L, Cheng S, Li H, Jiang J, Geng M, Jin Y and $\mathrm{Wu}$ Y: Expression and regulatory function of miRNA-34a in targeting survivin in gastric cancer cells. Tumour Biol 34: 963-971, 2013
28. Hagman Z, Haflidadottir BS, Ansari M, Persson M, Bjartell A, Edsjö A and Ceder Y: The tumour suppressor miR-34c targets MET in prostate cancer cells. Br J Cancer 109: 1271-1278, 2013.

29. Li N, Fu H, Tie Y, Hu Z, Kong W, Wu Y and Zheng X: miR-34a inhibits migration and invasion by downregulation of c-Met expression in human hepatocellular carcinoma cells. Cancer Lett 275: 44-53, 2009.

30. Kumar B, Yadav A, Lang J, Teknos TN and Kumar P: Dysregulation of microRNA-34a expression in head and neck squamous cell carcinoma promotes tumor growth and tumor angiogenesis. PLoS One 7: e37601, 2012.

31. Shen Z, Zhan G, Ye D, Ren Y, Cheng L, Wu Z and Guo J: MicroRNA-34a affects the occurrence of laryngeal squamous cell carcinoma by targeting the antiapoptotic gene survivin. Med Oncol 29: 2473-2480, 2012.

32. Wen J, Zhao YK, Liu Y and Zhao JF: MicroRNA-34a inhibits tumor invasion and metastasis in osteosarcoma partly by effecting C-IAP2 and Bcl-2. Tumour Biol 39: 1010428317705761, 2017.

33. Li L, Yuan L, Luo J, Gao J, Guo J and Xie X: miR-34a inhibits proliferation and migration of breast cancer through downregulation of Bcl-2 and SIRT1. Clin Exp Med 13: 109-117, 2013.

(i) $(5)$ This work is licensed under a Creative Commons Attribution-NonCommercial-NoDerivatives 4.0 International (CC BY-NC-ND 4.0) License. 